\title{
Comparison of IgM enzyme linked immuno sorbent assay with Weil-felix agglutination test for diagnosis of Scrub typhus in South India
}

\author{
Meerah Srinivasagam¹, Vijayalakshmi Arumugam², Rajeswari Jayakumar ${ }^{3, *}$ \\ 1, $\mathbf{3}^{*}$ Assistant Professor, ${ }^{2}$ Professor, Dept. of Microbiology, Chengalpattu Medical College, Chengalpattu, Tamil Nadu
}

*Corresponding Author:

Email: rajeswari1962@yahoo.co.in

\begin{abstract}
Introduction: Scrub typhus is a disease caused by Orientia (Rickettsia) tsutsugamushi which belongs to Rickettsiaeceae family. Early diagnosis of Scrub typhus is important because timely anti-microbial therapy may help an excellent response and prevent complications. Serology is widely used for diagnosis of scrub typhus. The Aim of this study isto detect the IgM antibodies by Capture Enzyme linked immuno sorbent assay (ELISA) and Weil -Felix agglutination test against Scrub typhus and to compare the results of IgM ELISA with Weil - Felix test.

Materials and Methods: Total of 723 blood samples were collected from clinically suspected Scrub typhus patients. IgM ELISA (commercial) and Weil -Felix agglutination test for Scrub typhus were performed on all serum samples.

Results: Among 723 patients, 31(4.2\%) showed positive by Weil-Felix test against OX K antigen and 43 (6\%) showed positive by IgM ELISA. Among 43samples which were positive by IgM ELISA, 13(1.8 \%) showed negative by Weil-Felix test. Out of 31 samples positive by Weil-Felix test, one sample showed negative by IgM ELISA. The sensitivity of IgM ELISA and Weil -Felix agglutination test is $96.77 \%$ and $69.76 \%$ respectively. Conclusion:Early diagnosis and treatment will prevent complications of Scrub typhus. This study has found out the IgM ELISA is highly sensitive and dependable for diagnosis of scrub typhus than Weil -Felix agglutination test.
\end{abstract}

Keywords: IgM Enzyme linked immuno sorbent assay, Scrub typhus, Weil Felix agglutination Test.

\section{Introduction}

Scrub typhus (mite-borne typhus, Tsutsugamushi disease) is an acute, febrile, infectious illness that is caused by Orientia (Rickettsia) tsutsugamushi which belongs to Rickettsiaeceae family. It was first described from Japan in 1899.It is a zoonotic disease, human are accidental hosts. $O$. tsutsugamushi includes heterogeneous strains classified in five major serotypes: Boryon, Gilliam, Karp, Kato and Kawazaki. ${ }^{7}$

Therefore surveillance is essential for a better understanding of the epidemiology of the disease and for the detection of outbreaks. An estimated one million casesoccur annually and mortality rates in untreated patients range from $0-30 \%$, secondary complications like MODS, pneumonitis, ARDS, encephalitis and circulatory failure. ${ }^{2}$ The Scrub typhus appears particularly to be distributed in the Tsutsugamushi triangle. The distribution is over a very wide area of 13 million Km square bound by Japan in the east, through China, the Philippines, tropical Australian the south and west through India, Pakistan possibly from Tibet to Afghanistan and southern parts of USSR in the north. The distribution of the disease closely follows the distribution of Rattusrattus. ${ }^{8}$ The vector of scrub typhus is present in most countries of the South-East Asia Region and it is endemic in certain geographical regions of India, Indonesia, Maldives, Myanmar, Nepal, Sri Lanka and Thailand.Scrub typhus is considered as emerging infectious disease in India

Usually it is an occupational disease in rural workers and can occur in all the age groups, particularly adults involved in agriculture and forest occupations and those who are in contact with the chiggers. Soldiers living in temporary camps are also predisposed to scrub typhus. It occurs more frequently during the rainy season. However Outbreaks of scrub typhus are reported in southern India during the colder months of the year. ${ }^{5}$ People of all ages are affected by it.

Prompt antibiotic therapy shortens the course of the disease, lowers the risk of complications and in turn reduces morbidity and mortality due to rickettsial diseases. Untreated cases have fatality rates as $30-45$ percent with multiple organ dysfunction, if not promptly diagnosed and appropriately treated. The vast variability and non-specific presentation of this infection have often made it difficult to diagnose clinically. Serological test are widely used for diagnosing scrub typhus.

\section{Materials and Methods}

The study was a retrospective study. Total of 723 blood samples were collected from clinically suspected Scrub typhus patients over the period of one year. The study was conducted at, a tertiary care hospital, South India.

IgM Enzyme linked immuno sorbent assay and Weil -Felix agglutination test for Scrub typhus were performed on all serum samples by usingcommercial IgM Capture ELISA kit (INBIOS, USA) and quantitative detection $O$. tsutsugamushi antibodies by using Proteus mirabilis OXK antigenby Weil -Felix agglutination test. IgM ELISA (Inbios, commercial kit) cut off value $>0.500$ considered as reactive for scrub typhus and Weil-Felix Agglutination titers of $\geq 160$ to OX K antigen were taken as positive for scrub typhus. 
The results of IgM Capture ELISA with Weil - Felix agglutination test was compared.

\section{Results}

Among 723 patients, and $43(6 \%)$ showed positive by IgM ELISA and 31(4.2\%) showed positive by WeilFelix test against OX K antigen (Table -1). Among 43 samples which were positive by IgM ELISA, 13 (1.8\%) showed negative by Weil-Felix test. Out of 31 samples positive by Weil-Felix test, one sample showed negative by IgM ELISA.

Table 2 shows that 31 samples positive for Weil Felix agglutination test, 2 samples $(6 \%)$ with a titre of $1: 1280,3$ samples $(10 \%)$ with a titre of $1: 640,12$ samples $(39 \%)$ with a titre of $1: 320$, and 14 samples $(45$ $\%$ ) with a titre of $1: 160$ If, the titre of $\geq 1: 160$ was considered as significant.

Female patients (28cases) were most commonly affected than male. Positivity was higher in the age group of patients between 31 and $50 \mathrm{yrs}$ of age.

Table 1: Positivity of scrub typhus by IgM capture elisa and weil - felix test $(\mathrm{n}=723)$

\begin{tabular}{|c|c|c|}
\hline S.N. & Test & No. of positive samples \\
\hline 1 & Total elisa positive & $43(6 \%)$ \\
\hline 2 & Total weil- felix test & $31(4.2 \%)$ \\
\hline
\end{tabular}

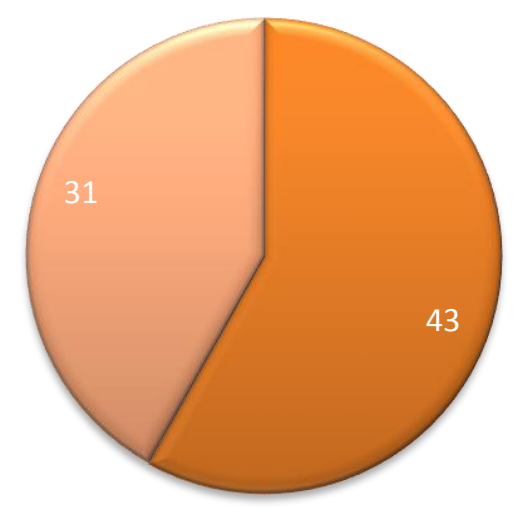

m Total IgM ELISA Positve

Fig. 1: Positivity of Scrub typhus by IgM ELISA and Weil - felix test

Table 2: Weil- felix test positivity with titre $(n=31)$

\begin{tabular}{|l|l|c|}
\hline S. N. & \multicolumn{1}{|c|}{ Significant titre } & No. of positive samples \\
\hline 1 & $>1: 1280$ & $2(6 \%)$ \\
\hline 2 & $1: 640$ & $3(10 \%)$ \\
\hline 3 & $1: 320$ & $12(39 \%)$ \\
\hline 4 & $1: 160$ & $14(45 \%)$ \\
\hline & TOTAL & $31(100 \%)$ \\
\hline
\end{tabular}

Table 3: Comparison of Weil- felix test with IgM ELISA

\begin{tabular}{|l|c|c|c|}
\hline & IgM ELISA Positive & IgM ELISA Negative & Total \\
\hline Weil- Felix Test Positive & $30(\mathrm{a})$ - True positive & $1(\mathrm{~b})-$ False positive & $31(\mathrm{a}+\mathrm{b})$ \\
\hline Weil- Felix Test Negative & $13(\mathrm{c})-$ False negative & $679(\mathrm{~d})-$ True negative & $692(\mathrm{c}+\mathrm{d})$ \\
\hline Total & $43(\mathrm{a}+\mathrm{c})$ & $680(\mathrm{~b}+\mathrm{d})$ & $723(\mathrm{a}+\mathrm{b}+\mathrm{c}+\mathrm{d})$ \\
\hline
\end{tabular}

Sensitivity $=$ True positive $/$ True positive + False negative $\times 100=a / a+c \times 100=30 / 43 \times 100=69.76 \%$. Specificity $=$ True negative $/$ False positive + True negative $\times 100=d / b+d \times 100=679 / 680 \times 100=99.85 \%$. Positive Predictive Value $=$ True positive $/$ True positive + False positive $\times 100=a / a+b \times 100=30 / 31 \times 100=96.77$ $\%$. 
Table 4: Comparision of IgM ELISA with Weil- felix test

\begin{tabular}{|l|c|c|c|}
\hline \multicolumn{1}{|c|}{ ELISA } & Weil- felix test positive & $\begin{array}{c}\text { Weil- felix test } \\
\text { negative }\end{array}$ & Total \\
\hline IgM ELISA positive & $30(\mathrm{a})-$ True positive & $13(\mathrm{~b})-$ False positive & $43(\mathrm{a}+\mathrm{b})$ \\
\hline IgM ELISA negative & $1(\mathrm{c})-$ False negative & $679(\mathrm{~d})-$ True negative & $680(\mathrm{c}+\mathrm{d})$ \\
\hline TOTAL & $31(\mathrm{a}+\mathrm{c})$ & $692(\mathrm{~b}+\mathrm{d})$ & $723(\mathrm{a}+\mathrm{b}+\mathrm{c}+\mathrm{d})$ \\
\hline
\end{tabular}

Sensitivity $=$ True positive $/$ True positive + False negative $\times 100=a / a+c \times 100=30 / 31 \times 100=96.77 \%$.

Specificity $=$ True negative $/$ False positive + True negative $\times 100=d / b+d \times 100=679 / 692 \times 100=98.12 \%$.

Positive Predictive Value $=$ True positive $/$ True positive + False positive $\times 100=\mathrm{a} / \mathrm{a}+\mathrm{b} \times 100=30 / 43 \times 100=69.76$ $\%$.

\section{Discussion}

Total of 723 blood samples were collected from clinically suspected Scrub typhus patients over the period of one year in a suburban area.

Female patients $(28 / 43$ cases, $65.1 \%)$ were most commonly affected than male.This study was correlates with the study done by PK Sharma et $\mathrm{al}^{6}$ and SS Kweon et $\mathrm{al}^{4}$ which showed female preponderance of $54.8 \%$ and $64.5 \%$ respectively. This female preponderance is due to the visit of most of the patients from rural area.

Positivity was higher in the age group of patients between 31 and $50 \mathrm{yrs}$ of age. This is concordance to the study done by PK Sharma et al. ${ }^{6}$ This is because of the active age group and many of them has agriculture as occupation.

IgM Enzyme linked immuno sorbent assay and Weil -Felix agglutination test for Scrub typhus were performed on all serum samples by usingCommercial IgM Capture ELISA kit (INBIOS, USA) and quantitative detection of $O$. tsutsugamushi antibodies by using Proteus mirabilis OXK antigenby Weil -Felix agglutination test. . IgM ELISA (Inbios, commercial kit) cut off value $>0.500$ considered as reactive for scrub typhus and Weil-Felix Agglutination titers of $\geq 160$ to OX K antigen were taken as positive for scrub typhus.

Among 723 samples, and $43(6 \%)$ showed positive by IgM ELISA and 31(4.2\%) showed positive by WeilFelix test against OX K antigen (Table 1). Among 43 samples which were positive by IgM ELISA, $13(1.8 \%)$ showed negative by Weil-Felix test. Out of 31 samples positive by Weil-Felix test, 1sample showed negative by IgM ELISA

Among the 31 samples positive for Weil - Felix agglutination test, 2 samples( $6 \%$ ) had a titre of 1:1280, 3 samples $(10 \%)$ with a titre of $1: 640,12$ samples(39\%) with a titre of $1: 320$, and 14 samples $(45 \%)$ with a titre of $1: 160$, the titre of $\geq 1: 160$ was considered as significant.

From the above results when compared to IgM capture ELISA, Weil-Felix agglutination test results may be negative during the early stages of the disease because agglutinating antibodies are detectable only during the second week of illness. ${ }^{1} \mathrm{We}$ found that the Weil- Felix agglutination test had $69.76 \%$. Sensitivity and $99.85 \%$ specificity and $96.77 \%$ positive predictive value at a titre of $\geq 1$ : 160. But IgM ELISA test, provides positive results within 3-4 days after the onset of illness and had
$96.77 \%$ sensitivity, $98.12 \%$ specificity and 69.76 $\%$.positive predictive value. IgM ELISA is more sensitive $(96.77 \%)$ than Weil- Felix agglutination test $(69.76 \%)$. According to Issac et al, Weil Felix test has high specificity, even at a titer as low as 1:20. ${ }^{3}$ Hence, they suggested that patients with low titres also should be evaluated for scrub typhus. However the test lacks sensitivity.

\section{Conclusion}

The Scrub typhus is one of the differential diagnosis of the acute febrile illnesses. It causes life threatening complications like renal failure, liver failure, ARDS, MODS, meningitis and thrombocytopenia. High index of suspicion and early diagnosis of scrub typhus by serological test can greatly reduce the chances of complications and can guide optimal therapy. The IgM capture ELISA is more sensitive test when compared to Weil- Felix agglutination test. But the Weil- Felix agglutination test is more specific though it shows sensitivity of $69.76 \%$ only.IgM ELISA for Scrub Typhus is more sensitive and rapid for testing large number of sera in early phase of disease. However, Weil - Felix agglutination test is more economical when compared to ELISA and it can be used in resource poor setting, where facility for ELISA and other tests are not available.

\section{References}

1. Amano K, Suzuki N, Hatakeyama H, et al. The reactivity between Rickettsiae and Weil-Felix test antigens against sera of rickettsial disease patients.ActaVirol1992;36:6772.

2. Dinesh Kumar, D.J. Raina, Sanjana Gupta, Ana Angurana. Epidemiology of Scrub Typhus.www.jkscience.org. Vol. 12 No. 2, April-June 2010.

3. Issac R, Varghese GM, Mathai E et al. Scrub Typhus: Prevalence and diagnostic issues in rural Southern India. Clin Infect Dis 2004;39:1395-6.

4. Kweon SS, Choi JS, Lim HS, Kim JR, Kim KY, Ryu SY, et al. Rapid increase of scrub typhus, South Korea, 20012006. Emerg Infect Dis 2009; 15: 1127-9.

5. Mathai E, Rolain JM, Verghese GM, et al. Outbreak of scrub typhus in southern India during the cooler months. Ann N Y AcadSci2003; 990:359-64.

6. Sharma PK, Ramakrishnan R, Hutin YJF, Barui AK, Manickam P, Kakkar M, et al. scrub typhus in Darjeeling, India: opportunities for simple, practical prevention measures. Trans R Soc Trop Med Hyg 2009; 103: 1153 8 . 
7. Tamura A, Ohashi N, Urakami H, Miyamura S, 1995.

Classification of Rickettsia tsutsugamushiin a new genus, Orientiagen.nov., as Orientiatsutsugamushicombnov.Int J SystBacteriol45:589-591.

8. World Health Organization Technical Report Series No.553 Ecology and control of Rodents of Public Health Importance. Report of a WHO Scientific Group.

How to cite this article: Srinivasagam M, Arumugam V, Jayakumar R. Comparison of IgM enzyme linked immuno sorbent assay with Weil-felix agglutination test for diagnosis of Scrub typhus in South India. Indian J Microbiol Res 2018;5(1):92-95. 